\title{
Down-sizing the overzealous search for low-risk thyroid malignancy
}

\author{
Laszlo Hegedüs ${ }^{1}$
}

Received: 13 January 2016/Accepted: 6 April 2016/Published online: 15 April 2016

(C) Springer Science+Business Media New York 2016

Questionnaires, albeit performed around a decade ago, illustrate considerable intercontinental, regional, and medical specialty-related differences in the management of nodular goiter [1]. This is most pronounced concerning the therapy options, once a diagnosis of benignity has been made. Thus, many advocate use of levothyroxine despite lack of its efficacy. Also many offer costly periodic followup without therapy. This contrasts the high overall agreement, as evident in recent guidelines [2]. Just a few decades ago, very few endocrinologists used ultrasound of the thyroid, even fewer performed fine-needle aspiration cytology (FNAC), or understood the implications of diagnoses made by FNAC. We lacked confidence in ruling out malignancy by FNAC, abused diagnostic thyroidectomy, and lacked understanding of the natural course of supposedly benign thyroid nodules. Worst of all, we neglected understanding the implications of offering a diagnosis which implies a certain risk of overlooking malignancy.

Is the increasing availability of ultrasound investigations, and its liberal use, then a problem? The diagnosis of increasing numbers of individuals with thyroid nodules, and a correspondingly higher number of mostly low-risk papillary thyroid cancers, suggests this to be factual and has-perhaps counterintuitively — not changed these individuals' prognosis. The most recent evidence of the consequences of early detection of thyroid nodules and malignancies come from South Korea [3]. Here, over two decades (1993-2011), the rate of thyroid cancer diagnoses increased 15 -fold, with unaltered mortality rates. Similar

Laszlo Hegedüs

Laszlo.hegedus@rsyd.dk

1 Department of Endocrinology and Metabolism, Odense University Hospital, 5000 Odense C, Denmark tendencies have been reported from, e.g., Europe and the USA. Therefore, although we lack exact numbers, the additional cost to society of diagnosing and following such individuals is tremendous. Accepting that the anxiety of either superfluous surgery or being reminded of still harboring a nodule remains to be explored, this may well overrule the benefit of early diagnosis in a few individuals with high-risk malignancy. It is therefore not surprising that so much effort has gone into cost-effective algorithms for diagnosis and therapy $[4,5]$, aiming at reducing FNAC and allowing release of patients from followup, based on low risk of overlooking malignancy.

Reinforcing this line of thought, Burman and Wartofsky, in a recent New England Journal of Medicine review [5], give an account of the central aspects of thyroid nodule management. They maintain the central role of ultrasoundguided FNAC, and that cytology suspicious for malignancy should in general lead to referral for thyroidectomy. In line with others [6], they also focus on the unclarified role of molecular testing to rule out malignancy when cytology is suspicious. Even when sampling is correct, the negative predictive value is no higher than $95 \%$. Whether this allows conservative monitoring-as suggested by the authors [5] - is highly dependent on a multitude of factors including local legislation and is not shared by all [6].

Another issue is whether nodule size should influence the recommendation of FNAC. While FNAC is often recommended for nodules $>1-2 \mathrm{~cm}$ in greatest dimension, there is no clear-cut correlation between increasing size and risk of malignancy. Neither is there consensus on interval of followup in patients with benign cytology. In this light, it is interesting that two papers, in recent issues of Endocrine, focus on these aspects. Thus, Brito et al. [7], in a population-based but selected group of patients from Olmsted County, Minnesota, USA, investigated 485 
thyroid nodules (46; $9.5 \%$ with thyroid cancer). Of these cancers, only eight $(1.6 \%)$ and one $(0.2 \%)$ had American Thyroid Association intermediate risk and high-risk scores, respectively. Thyroid cancer frequency and risk scores were evenly distributed across tumor sizes. Of the 105 nodules $<1 \mathrm{~cm}$, only 13 were malignant, of which only 2 were classified as intermediate risk and none with high-risk features. No case of persistent disease occurred over a median followup of 7 years. While avoiding cytology in tumors $<1 \mathrm{~cm}$ would not miss high-risk thyroid cancer, it is also clear that tumor size could not be used as a surrogate for accurately differentiating between ATA risk scores. Kim et al., also in this issue [8], in a large-scale retrospective study of selected patients with thyroid nodules sized 5-10 mm, followed 378 patients (447 nodules) for a minimum of 3 years. Seven malignancies $(1.6 \%)$ were detected after a mean followup of 70 months (range 36-104 months). Only one demonstrated growth $>3 \mathrm{~mm}$ and none showed signs of extrathyroidal growth or signs of metastasizing, and all were papillary thyroid microcarcinomas. The authors conclude that initial FNAC, in such patients, is unwarranted and could be replaced by an ultrasound investigation after 3 years [8]. This recommendation concords well with those in the recent 2015 American Thyroid Association Guidelines [9], in that FNAC in nodules $<1 \mathrm{~cm}$ should be avoided, and that risk assessment should be based on ultrasound features before biopsy to avoid unnecessary biopsies. At least for Europeans faced with the consequences of iodine deficiency, none of these papers deal with the much more common, and cumbersome, issue of multinodular goiter, which, diagnostically and therapeutically, offers more challenge [10].

In the end, an algorithm ideal for the virtual patient is always replaced by thoughtful dialog between the caretaking physician and the patient. Thus, two identical patient situations may be dealt with differently. However, and as emphasized by the data of Brito et al. [7] and Kim et al. [8], our overzealous attempts to diagnose thyroid malignancy may have harmed many patients and prioritized our resources inadvertently/erroneously. Statistically, the risk of overdiagnosis, superfluous FNAC, unnecessary surgery, or followup is much higher than the risk of overlooking malignancy that will negatively impact the prognosis or quality of life of our patients. This needs to be borne in mind when attempting a balance between postponing the diagnosis of low-risk malignancy and prioritizing our resources. Here, we and our legislators need to accept that postponing the diagnosis of low-risk malignancy poses little threat to our patients. Thus, to follow up on the title, down-sizing the search for low-risk thyroid malignancy, by up-sizing the criteria for which thyroid nodules get scrutinized, is warranted. However, despite such endeavors, current efforts to deflate cost of thyroid nodule management will only alter the slope of increase. In order genuinely to "turn the tide" [11], increased physician, public, and legislative awareness of the consequences of current management needs to be paired with focus on more accurate characterization of thyroid nodules. Should we fail in this reversal of management [12], our successors may rightfully blame us for negligence.

Acknowledgments None.

Compliance with ethical standards

Conflict of interest None.

\section{References}

1. J.P. Walsh, S.A. Ryan, D. Lisewski, M.Z. Alhamoudi, S. Brown, F.N. Bennedbaek, L. Hegedüs, Differences between endocrinologists and endocrine surgeons in management of the solitary thyroid nodule. Clin. Endocrinol. 66(6), 844-853 (2007). doi:10. 1111/j.1365-2265.2007.02823.x

2. R. Paschke, L. Hegedüs, E. Alexander, R. Valcavi, E. Papini, H. Gharib, Thyroid nodule guidelines: agreement, disagreement and need for future research. Nat. Rev. Endocrinol. 7(6), 354-361 (2011). doi:10.1038/nrendo.2011.1

3. H.S. Ahn, H.J. Kim, H.G. Welch, Korea's thyroid-cancer "epidemic"-screening and overdiagnosis. N. Engl. J. Med. 371, 1765-1767 (2014). doi:10.1056/NEJMp1409841

4. L. Hegedüs, The thyroid nodule. N. Engl. J. Med. 351, 1764-1771 (2004). doi:10.1056/NEJMcp031436

5. K.D. Burman, L. Wartofsky, Thyroid nodules. N. Engl. J. Med. 373, 2347-2356 (2015). doi:10.1056/NEJMcp1415786

6. M. Eszlinger, L. Hegedüs, R. Paschke, Ruling in or ruling out thyroid malignancy by molecular diagnostics of thyroid nodules. Best Pract. Res. Clin. Endocrinol. Metab. 28(4), 545-557 (2014). doi:10.1016/j.beem.2014.01.011

7. J.P. Brito, N. Singh-Ospina, M.R. Gionfriddo, S. Maraka, A. Espinosa De Ycaza, R. Rodriguez-Gutierrez, J.C. Morris, V.M. Montori, R.M. Tuttle, Restricting ultrasound thyroid fine needle aspiration biopsy by nodule size: which tumors are we missing? A population-based study. Endocrine. 51(3), 499-505 (2016). doi:10.1007/s12020-015-0713-8

8. S.-Y. Kim, H.S. Lee, E.-K. Kim, H.J. Moon, J.H. Yoon, J.H. Hong, J.Y. Kwak, Follow-up ultrasound may be enough for thyroid nodules from $5 \mathrm{~mm}$ to $1 \mathrm{~cm}$ in size. Endocrine 52(1), 130-138 (2016). doi:10.1007/s12020-015-0740-5

9. B.R. Haugen, E.K. Alexander, K.C. Bible, G.M. Doherty, S.J. Mandel, Y.E. Nikiforov, F. Pacini, G.W. Randolph, A.M. Sawka, M. Schlumberger, K.G. Schuff, S.I. Sherman, J.A. Sosa, D.L. Steward, R.M. Tuttle, L. Wartofsky, 2015 American Thyroid Association management guidelines for adult patients with thyroid nodules and differentiated thyroid cancer: The American Thyroid Association guidelines task force on thyroid nodules and 
differentiated thyroid cancer. Thyroid 26(1), 1-133 (2016). doi:10.1089/thy.2015.0020

10. L. Hegedüs, S.J. Bonnema, F.N. Bennedbaek, Management of simple nodular goiter: current status and future perspectives. Endocr. Rev. 24(1), 102-132 (2003). doi:10.1210/er.2002-0016

11. H.S. Ahn, H.G. Welch, South Korea's thyroid-cancer "epidemic"-turning the tide. N. Engl. J. Med. 373, 2389-2390 (2015). doi:10.1056/NEJMc1507622
12. V.K. Prasad, A.S. Cifu, Ending Medical Reversal: Improving Outcomes, Saving Lives (John Hopkins University Press, Baltimore, 2015). ISBN 978-1-4214-1772-1 